\title{
Evaluación de Dos Crioprotectores y Tres Curvas de Congelación Programable en la Criopreservación de Semen de Brycon henni (Pisces: Characidae)
}

\author{
Evaluation of Two Cryprotectans and Three Curves of Programable Freezing in \\ Semen Cryopreservation of Brycon henni (Pisces: Characidae)
}

Giovanni Restrepo-Betancur ${ }^{1,3}$, Juan David Montoya Páez $^{2}$, Lucy Arboleda Chacón ${ }^{2}$

\section{Resumen}

\begin{abstract}
El objetivo del presente estudio fue evaluar la criopreservación de semen de Brycon henni, mediante tres curvas de congelación programable y con el empleo de dos crioprotectores permeables. El semen de 80 machos se diluyó en un medio suplementado con etilenglicol (EG) o dimetilsulfóxido (DMSO) y se crioconservó en pajillas de semen, utilizando tres curvas de congelación programable: lenta $\left(66 \mathrm{~min},-0.42^{\circ} \mathrm{C} / \mathrm{min}\right)$, media (43.3 $\mathrm{min},-0.6^{\circ} \mathrm{C} / \mathrm{min}$ ) y ultra-rápida $\left(7.7 \mathrm{~min},-5.19^{\circ} \mathrm{C} / \mathrm{min}\right)$. Después de un mes de almacenamiento, el semen fue descongelado y se evaluó la movilidad, mediante el sistema de análisis de clase $\left(\mathrm{SCA}^{\circledR}\right)$ y la vitalidad espermática (VE) mediante microscopía de fluorescencia con las sondas SYBR14/IP. Para el análisis estadístico se ajustaron modelos lineales generalizados (GLM) y las medias se compararon por la prueba de Tukey. Las curvas de velocidad media y ultra-rápida presentaron valores superiores y equivalentes para la movilidad total, la movilidad progresiva y la velocidad lineal de los espermatozoides $(\mathrm{p}<0.05)$. La vitalidad espermática fue superior empleando la curva de velocidad media $(52.4 \pm 8.6 \%)$ en relación a las curvas ultra-rápida $(43.0 \pm 19.4 \%)$ y lenta $(29.0 \pm 11.8 \%)$ $(\mathrm{p}<0.05)$. El EG presentó una mejor vitalidad espermática $(\mathrm{p}<0.05)$. Se concluye que la congelación de semen de sabaleta $(B$. henni) usando una curva de congelación programable de velocidad media y etilenglicol como crioprotector permite resultados superiores de calidad seminal posdescongelación.
\end{abstract}

Palabras clave: criopreservación; congelación programable; peces; espermatozoide; sabaleta

\footnotetext{
${ }^{1}$ Facultad de Ciencias Agrarias, Universidad Nacional de Colombia, Medellín, Colombia

${ }^{2}$ Facultad de Ciencias Agrarias, Politécnico Colombiano Jaime Isaza Cadavid, Medellín, Colombia

${ }^{3}$ E-mail: jdmontoya@elpoli.edu.co
}

Investigación financiada y ejecutada mediante el convenio 4600000980 entre la Secretaria de Agricultura y Desarrollo Rural del Departamento de Antioquia y el Politécnico Colombiano Jaime Isaza Cadavid

Recibido: 13 de setiembre de 2016

Aceptado para publicación: 18 de marzo de 2017 
The aim of this study was to evaluate the cryopreservation of Brycon henni semen using three programmable freezing curves and two permeable cryoprotectants. Semen of 80 males were diluted in medium supplemented with ethylene glycol (EG) or dimethylsulfoxide (DMSO), and cryopreserved in semen straws using three programmable freezing curves: slow $\left(66 \mathrm{~min},-0.42^{\circ} \mathrm{C} / \mathrm{min}\right)$, medium $\left(43.3 \mathrm{~min},-0.6^{\circ} \mathrm{C} / \mathrm{min}\right)$ and ultra-rapid ( $7.7 \mathrm{~min},-5.19^{\circ} \mathrm{C} / \mathrm{min}$ ). After one month of storage, semen was thawed and motility was evaluated through a class analysis system $(\mathrm{SCA} \AA)$ and sperm vitality $(\mathrm{EV})$ by fluorescence microscopy with SYBR14/IP probes. For statistical analysis, generalized linear models (GLM) were adjusted and means compared by the Tukey test. The medium and ultrarapid velocity curves showed higher and equivalent values for total motility, progressive motility, and linear velocity of sperm $(\mathrm{p}<0.05)$. Sperm vitality was better in the medium velocity curve $(52.4 \pm 8.6 \%)$ in comparison with the ultra-rapid $(43.0 \pm 19.4 \%)$ and slow $(29.0 \pm 11.8 \%)$ curves $(\mathrm{p}<0.05)$. EG showed greater sperm vitality $(\mathrm{p}<0.05)$. It is concluded that freezing of sabaleta semen $(B$. henni) by a medium velocity programmable freezing curve and ethylene glycol as cryoprotectant allows better results of post-thaw semen quality.

Key words: cryopreservation; programmable freezing; fish; spermatozoa; sabaleta

\section{INTRODUCCIÓN}

La criopreservación ha facilitado la reproducción artificial en peces, debido a que mantiene la viabilidad del esperma almacenado en nitrógeno líquido por largo tiempo (Viveiros y Godinho, 2009). Esta técnica permite la mejora de la eficiencia del uso de parentales y la disminución de la presión sobre las poblaciones silvestres (Medina et al., 2005). Sin embargo, la criopreservación de semen de peces es un campo relativamente nuevo, donde se ha avanzado poco a nivel comercial, pese a que ya se dispone de protocolos de criopreservación para diversas especies (Carneiro et al., 2012). En Colombia, los estudios de criopreservación de semen de peces nativos son recientes y se han orientado principalmente a la estandarización de protocolos de congelación, evaluación de crioprotectores y diluyentes para disminuir los efectos tóxicos y el daño criogénico sobre la célula espermática (Martínez et al., 2011).
Brycon henni (Eigenmann 1913), orden Characiformes, familia Characidae, subfamilia Bryconinae, comúnmente conocida como sabaleta, es una de las especies migratorias de importancia en los ríos pequeños que atraviesan las zonas cafeteras de Colombia, y cuyo origen se encuentra en la cordillera central del país (Pineda-Santis et al., 2007). Las poblaciones naturales de sabaleta están decreciendo por la sobrepesca, la contaminación de su hábitat y la fragmentación de las corrientes por la construcción y operación de represas (Lenis et al., 2009).

La especie presenta dos épocas reproductivas en el año (Martínez et al., 2001; Arboleda et al., 2005), registrándose discrepancias en las épocas del año para el desove, el cual puede ocurrir durante el periodo de mayor precipitación o en otra época del año (Montoya et al., 2006). Se ha sugerido que existe asincronía entre los picos de madurez de hembras y machos a lo largo del año, sien- 
do más prolongada la época reproductiva en los machos (Martínez y Vásquez, 2001; Montoya et al., 2006).

Se requiere establecer protocolos eficientes de conservación que garanticen la disponibilidad de material genético de la especie con el fin de poder utilizarlo en programas de conservación, repoblamiento y producción comercial. En un trabajo reciente, se reportó la criopreservación de semen de Brycon henni, mediante la evaluación de crioprotectores bajo un protocolo de congelación convencional (Pineda-Santis et al., 2015). El objetivo de esta investigación fue evaluar la criopreservación de semen de Brycon henni, mediante tres curvas de congelación programable con dos crioprotectores permeables.

\section{Materiales y Métodos}

\section{Colección de Semen}

Se seleccionaron 80 machos Brycon henni sexualmente maduros del Centro Experimental y de Producción Acuícola CEPA - (06²6’37.57" N, 7544’04.93" W) en San Jerónimo (Antioquia, Colombia), el cual es parte del Politécnico Colombiano Jaime Isaza Cadavid.

Los peces se sometieron a condiciones similares de alimentación, ambiente, manejo productivo y reproductivo. Se realizaron tres colectas seminales entre mayo y septiembre de 2015, donde la pluviosidad fue de 185, $101 \mathrm{y}$ $96 \mathrm{~mm} / \mathrm{mes}$, respectivamente. Para la colección del semen, los peces se anestesiaron con una dosis de $30 \mathrm{mg} / 1$ de MS-222 ${ }^{\circledR}$ (Western Chemical, EEUU) y se colocaron sobre una toalla húmeda. Se les cubrió la cabeza, se retiró el exceso de agua de la zona ventral alrededor del poro genital y mediante una leve presión cráneo caudal, se procedió a la extracción y recolección del semen en un tubo de $1.5 \mathrm{ml}$. El tiempo promedio de cada colecta fue de $10 \mathrm{seg}$, aproximadamente.
Se evaluó la activación espermática al contacto con el agua de los estanques mediante microscopía (Eclipse E200, Nikon, Japón) para garantizar la viabilidad de cada muestra. Las muestras individuales fueron suspendidas en proporción 1:5 en un diluyente a base de leche semidescremada, azucares y antibióticos. El semen se transportó al Laboratorio de Biotecnología Animal del Politécnico Colombiano Jaime Isaza Cadavid, ubicado en Bello, Antioquia (Colombia), dentro de las dos horas posteriores a la colecta, $\mathrm{y}$ bajo condiciones de refrigeración $\left(5^{\circ} \mathrm{C}\right)$.

Los peces se manejaron según las directrices publicadas para la experimentación de peces en las Buenas Prácticas en la Producción Acuícola (ICA, 2007).

\section{Criopreservación de Semen}

En cada proceso se colectaron 15 muestras seminales, se mezclaron y luego se dividieron en tres alícuotas. Cada alícuota se asignó aleatoriamente a una de tres curvas de congelación programable: lenta $\left(66 \mathrm{~min},-0.42{ }^{\circ} \mathrm{C} /\right.$ $\mathrm{min})$, media (43.3 $\left.\mathrm{min},-0.6^{\circ} \mathrm{C} / \mathrm{min}\right)$ y ultra-rápida $\left(7.7 \mathrm{~min},-5.19^{\circ} \mathrm{C} / \mathrm{min}\right)$. Cada alícuota fue subdividida en dos, las cuales fueron suplementadas con los crioprotectores permeables (CP) dimetilsulfóxido (DMSO) o etilenglicol (EG).

La dilución se realizó con el diluyente base suplementado con $5 \%$ de cada CP y $5 \%$ de yema de huevo, en cantidad suficiente para una concentración final de espermatozoides de $1000-2000 \times 10^{6} / \mathrm{ml}$. La mezcla se mantuvo en refrigeración a $4{ }^{\circ} \mathrm{C}$ por 30 min y se empacaron en pajillas de 0.5 $\mathrm{ml}$. Luego, las pajillas se mantuvieron a $4{ }^{\circ} \mathrm{C}$ por 10 min adicionales antes de ser sometidas a las curvas de congelación en un congelador programable Crysalys Cryocontroller PTC-9500 (Biogenic, EEUU). Al final de cada curva de congelación, las pajillas se sumergieron en un tanque de nitrógeno líquido $\left(-196^{\circ} \mathrm{C}\right)$. 


\section{Evaluación Seminal Posdescongelación}

Las pajillas fueron descongeladas luego de 30 días de almacenamiento en nitrógeno líquido. Se evaluaron los parámetros de movilidad total (MT), movilidad progresiva (MP), velocidad curvilínea (VCL), velocidad lineal (VSL) y velocidad media (VAP). El semen fue evaluado mediante el sistema de análisis espermático asistido por computador Sperm Class Analizer (SCA ${ }^{\circledR}$, Microptic SL, España), mediante el procedimiento reportado por Pineda-Santis et al. (2015). Se empleó un microscopio de contraste de fase (Eclipse E200, Nikon, Japón) con una cámara digital (Scout SCA780, Basler, Alemania). Se estableció una configuración específica para el software de cámara de cubreobjetos de $20 \times 20 \mathrm{~mm}$, óptica en lente de ph-, gota de $7 \mu$, platina térmica a $37^{\circ} \mathrm{C}$ y un tamaño de partícula de 1 a $20 \mu \mathrm{m}$. En una cámara de Neubauer (para evitar el desplazamiento de la muestra) se mezcló $1 \mu 1$ de semen y $10 \mu 1$ de agua destilada.

La vitalidad espermática (VE) se evaluó con el kit Live/Dead (Molecular Probes, EEUU). Una suspensión de espermatozoides, en buffer fosfato con $1 \%$ de albúmina sérica bovina (BSA), fue incubada por $5 \mathrm{~min}$ a $35^{\circ} \mathrm{C}$ con el fluorocromo para ácidos nucleicos SYBR-14, a una concentración final de $6 \mathrm{mM}$. Luego los espermatozoides se incubaron de igual forma con el fluorocromo de ácidos nucleicos yoduro de propidio a $0.48 \mathrm{mM}$. Muestras de $5 \mu 1$ se utilizaron para el conteo de 200 células mediante un microscopio E200 con fluorescencia HBO (Nikon, Japón) (Gamboa et al., 2010).

\section{Evaluación Estadística}

Se utilizó un diseño completamente al azar. Los datos fueron analizados mediante modelos lineales generalizados (GLM) para las variables dependientes de calidad seminal. Se incluyeron los efectos fijos de mes de recolección (mayo, junio, septiembre), crioprotector permeable y curva de congela- ción, así como el efecto fijo de la interacción entre estas variables. Se evaluó la normalidad de los datos por la prueba K-S. Para la comparación de las medias ajustadas entre los tratamientos se realizó la prueba de Tukey. Todos los análisis fueron realizados mediante el programa SAS v. 9.2 (SAS Inst, Cary, NC).

\section{Resultados}

Los valores de calidad del semen fresco fueron para volumen de $350 \pm 176 \mu 1$, MT de $88.1 \pm 21.3 \%$, MP de $66.2 \pm 29.5 \%$, VCL de $107.7 \pm 37.3 \mu \mathrm{m} / \mathrm{s}$, VSL de $59.7 \pm 23.6$ $\mu \mathrm{m} / \mathrm{s}$ y VAP de $76.8 \pm 29.0 \mu \mathrm{m} / \mathrm{s}$. Los resultados de la evaluación de 70 pajillas de semen luego de la descongelación son explicados por los modelos estadísticos en una alta proporción para las variables dependientes MT, MP y VE $\left(\mathrm{R}^{2}>0.70\right)$, mientras que los parámetros cinéticos (VCL, VSL y VAP) fueron explicados en menor medida por los efectos incluidos $\left(\mathrm{R}^{2}=0.30-0.40\right)$.

En los modelos estadísticos para MT, MP y VE, los efectos del mes de recolección del semen, la curva de congelación y el efecto fijo de la interacción entre el mes de recolección, la curva de congelación y el crioprotector fueron significativos $(p<0.05)$. En los parámetros de cinética espermática no hubo significancia estadística de los efectos incluidos. Los coeficientes de variación (CV) para MT y MP fueron del 42.2 y $58.8 \%$, respectivamente, en tanto que el $\mathrm{CV}$ fue menor del $20 \%$ para los demás parámetros. El Cuadro 1 presenta los resultados para los parámetros de calidad seminal posdescongelación.

El efecto fijo del crioprotector no fue significativo para las variables en estudio; sin embargo, como se mencionó previamente, la interacción con el mes de recolección y la curva de congelación fue significativa $(p<0.05)$. Por otro lado, solo la VE fue estadísticamente diferente entre los crioprotectores $(p<0.05)$. El Cuadro 2 pre- 
Cuadro 1. Calidad espermática posdescongelación (media \pm desviación estándar) de semen de sabaleta Brycon henni crioconservado utilizando tres curvas de congelación programable

\begin{tabular}{lcccccc}
\hline Curva & MT & MP & VCL & VSL & VAP & VE \\
\hline Lenta & $15.9 \pm 9.4^{\mathrm{b}}$ & $2.9 \pm 2.4^{\mathrm{b}}$ & $42.0 \pm 5.9^{\mathrm{a}}$ & $22.7 \pm 6.9^{\mathrm{b}}$ & $31.5 \pm 6.8^{\mathrm{a}}$ & $29.0 \pm 11.8^{\mathrm{c}}$ \\
Media & $32.5 \pm 16.3^{\mathrm{a}}$ & $7.3 \pm 5.4^{\mathrm{a}}$ & $46.1 \pm 6.2^{\mathrm{a}}$ & $26.3 \pm 4.8^{\mathrm{a}}$ & $35.5 \pm 5.6^{\mathrm{a}}$ & $52.4 \pm 8.7^{\mathrm{a}}$ \\
Ultra-rápida & $29.2 \pm 21.6^{\mathrm{a}}$ & $5.8 \pm 5.5^{\mathrm{a}}$ & $43.4 \pm 6.0^{\mathrm{a}}$ & $24.4 \pm 4.2^{\mathrm{ab}}$ & $33.3 \pm 5.1^{\mathrm{a}}$ & $43.0 \pm 19.4^{\mathrm{b}}$ \\
\hline
\end{tabular}

Curvas de congelación programable: lenta (66 $\mathrm{min},-0.42 \mathrm{o} / \mathrm{min})$, media (43.3 $\mathrm{min},-0.6 \mathrm{o} / \mathrm{min}$ ) y ultra-rápida (7.7 $\mathrm{min},-5.19$ 일 $\mathrm{min}$ )

MT: Movilidad total (\%); MP: Movilidad progresiva (\%); VCL: Velocidad curvilínea ( $\mu \mathrm{m} / \mathrm{s})$; VSL: Velocidad lineal ( $\mu \mathrm{m} / \mathrm{s})$; VAP: Velocidad media $(\mu \mathrm{m} / \mathrm{s})$; VE: Vitalidad espermática (\%)

$a, b, c$ Letras diferentes denotan diferencia estadística $(p<0.05)$

Cuadro 2. Calidad espermática de semen de Brycon henni crioconservado (media \pm desviación estándar) con dimetilsulfóxido (DMSO) y etilenglicol (EG) como crioprotectores permeables

\begin{tabular}{ccccccc}
\hline $\begin{array}{c}\text { Crio- } \\
\text { protector }\end{array}$ & MT & MP & VCL & VSL & VAP & VE \\
\hline EG & $25.9 \pm 19.2^{\mathrm{a}}$ & $5.8 \pm 5.6^{\mathrm{a}}$ & $45.2 \pm 6.4^{\mathrm{a}}$ & $25.0 \pm 7.2^{\mathrm{a}}$ & $34.2 \pm 7.6^{\mathrm{a}}$ & $42.9 \pm 16.0^{\mathrm{a}}$ \\
$\mathrm{DMSO}$ & $25.6 \pm 16.5^{\mathrm{a}}$ & $4.8 \pm 4.2^{\mathrm{a}}$ & $42.2 \pm 5.7^{\mathrm{b}}$ & $23.8 \pm 3.3^{\mathrm{a}}$ & $32.5 \pm 4.0^{\mathrm{a}}$ & $39.6 \pm 17.8^{\mathrm{b}}$ \\
\hline
\end{tabular}

MT: Movilidad total (\%); MP: Movilidad progresiva (\%); VCL: Velocidad curvilínea ( $\mu \mathrm{m} / \mathrm{s})$; VSL: Velocidad lineal ( $\mu \mathrm{m} / \mathrm{s})$; VAP: Velocidad media $(\mu \mathrm{m} / \mathrm{s})$; VE: Vitalidad espermática (\%)

$a, b$ Letras diferentes denotan diferencia estadística $(p<0.05)$

senta los resultados de la evaluación espermática posdescongelación del semen criopreservado en presencia de EG o DMSO.

Las diferencias en la vitalidad espermática debidas al crioprotector permeable, según la curva de congelación empleada, se presentan en la Figura 1.

\section{Discusión}

Ese estudio permitió conocer la posibilidad de preservar semen de $B$. henni mediante un protocolo confiable y repetible de congelación programable. En el trabajo de Pineda-Santis et al. (2015), utilizando con- 


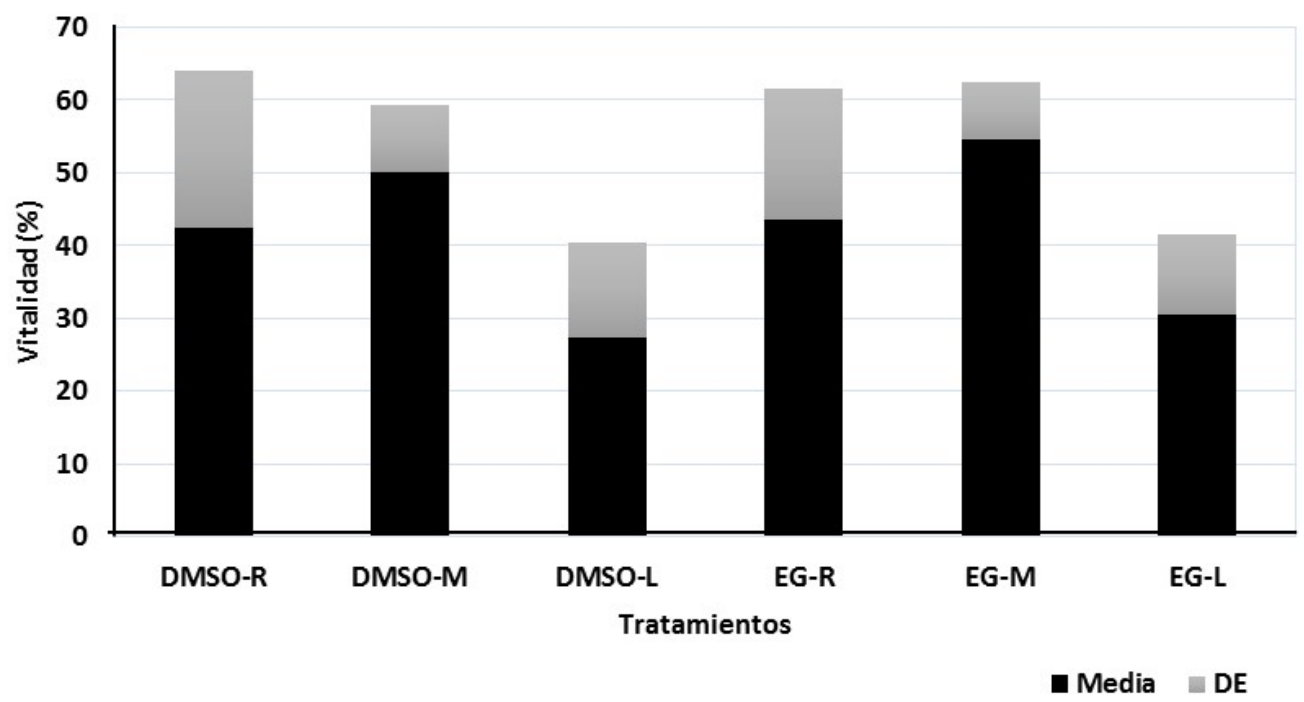

Figura 1. Vitalidad espermática de semen de Brycon henni crioconservado utilizando dos crioprotectores (DMSO: dimetilsulfoxido, EG: etilenglicol) y tres curvas de congelación (L: curva lenta ([6 $\left.\mathrm{min},-0.42^{\circ} \mathrm{C} / \mathrm{min}\right]$; M: curva media [43.3 $\left.\mathrm{min},-0.6^{\circ} \mathrm{C} / \mathrm{min}\right]$; $\mathrm{R}$ : curva ultra-rápida $\left[7.7 \mathrm{~min},-5.19^{\circ} \mathrm{C} / \mathrm{min}\right]$ )

gelación convencional se lograron resultados de calidad seminal superiores a los encontrados en esta investigación; lo cual podría ser debido al protocolo de congelación utilizado, donde pudieron diferir las tasas de descenso de temperatura entre los procedimientos por vapores de nitrógeno de dicho trabajo, versus los tratamientos de congelación programable de esta investigación. Otro factor determinante pudo ser la calidad de las muestras seminales utilizadas, la cual pudo presentar variaciones por efectos como la época y la edad de los reproductores. Se indica que la criopreservación de semen en peces presenta resultados altamente variables, dado que se trata de un proceso muy sensible a diversos factores, siendo necesario realizar ajustes específicos a los protocolos para cada especie (Medina et al., 2005; Martínez, 2008).

Factores como la velocidad de descenso de temperatura y los crioprotectores utilizados son determinantes en el éxito de la criopreservación seminal. El DMSO es uno de los crioprotectores permeables de bajo peso molecular más empleado en la criopreservación de semen de teleósteos tropicales, tales como yamú (Brycon siebenthalae), cachama blanca (Piaractus brachypomus) y bocachico (Prochilodus magdalenae) (Atencio et al., 2013). En esta investigación no se encontraron diferencias en la calidad seminal por efecto de los crioprotectores usados; sin embargo, en un estudio previo se había observado diferencias menores en la calidad del semen criopreservado de $B$. henni con EG y DMSO (Pineda-Santis et al., 2015). Cruz-Casallas et al. (2006) reportaron una MT de 76 y $60 \%$ con los crioprotectores DMSO al 5\% y EG al 5\%, respectivamente, para B. amazonicus. De otro lado, Carolsfeld et al. (2003) sugirieron que el DMSO al 10\% proporciona mejores resultados como crioprotector para los carácidos migratorios brasileros, entre los que se incluye el género Brycon. 
Dadas las escasas investigaciones existentes para la criopreservación seminal de $B$. henni, se hace necesario evaluar otras alternativas, como la combinación de crioprotectores y el modo de suplementación de otras sustancias protectoras. Babiak et al. (2001) encontraron un efecto benéfico en la combinación de crioprotectores permeables y yema de huevo como crioprotector no permeable, cuando crioconservaron semen de trucha arco iris (Oncorhynchus mykiss). De otro lado, María et al. (2006) observaron que la movilidad posdescongelación del semen de piracanjuba (B. orbignyanus) estuvo fuertemente correlacionada con el uso de la yema de huevo en la solución de congelación. De acuerdo con Gaitan-Espitia et al. (2013), los protocolos deben ser optimizados para cada una de las especies, de modo que se pueda mantener la calidad de los espermatozoides.

Los resultados de esta investigación muestran que una curva de congelación para el semen de $B$. henni, con una velocidad de descenso de temperatura de $-0.6^{\circ} \mathrm{C} / \mathrm{min}$ y un tiempo de 43 minutos, produce mejores resultados de calidad seminal posdescongelación que las curvas evaluadas con menor o mayor velocidad de descenso de temperatura, así como con menor o mayor tiempo total de exposición. Sin embargo, dada la gran posibilidad de opciones de configuración de una curva de congelación, es probable que pueda optimizarse aún más la congelación programable de semen para la especie.

En una investigación con semen de Pangasius sutchi se empleó una velocidad de descenso de temperatura de $-10{ }^{\circ} \mathrm{C} / \mathrm{min}$ y el crioprotector permeable DMSO con resultados de MT posdescongelación de 79.2 \pm 2.0\% (Rani et al., 2016). En otro estudio con semen de carpa plateada (Hypophthalmichthys molitrix) se utilizó una velocidad de descenso de temperatura de $-10{ }^{\circ} \mathrm{C} / \mathrm{min}$ entre $-4{ }^{\circ} \mathrm{C}$ y $-80^{\circ} \mathrm{C}$, con lo cual se logró una MT posdescongelación de $74 \pm 4.2 \%$ (Hossain y Sarder, 2009). De otro lado, en un trabajo de congelación de semen de carpa común (Cyprinus carpio) con DMSO al
$10 \%$, se realizó un descenso de $-5{ }^{\circ} \mathrm{C} / \mathrm{min}$ entre $20{ }^{\circ} \mathrm{C}$ y $-20{ }^{\circ} \mathrm{C}$ y posteriormente de $10{ }^{\circ} \mathrm{C} / \mathrm{min}$ entre $-20{ }^{\circ} \mathrm{C}$ y $-40{ }^{\circ} \mathrm{C}$, obteniéndose una MT de 78\% y una MP de 75.8\% (Rani y Munuswamy, 2014).

En la evaluación de la vitalidad se observó igualmente superioridad de la curva de velocidad media (Cuadro 1), al igual que del EG sobre el DMSO (Cuadro 2) $(\mathrm{p}<0.05)$. En el estudio de Pineda-Santis et al. (2015) EG fue igualmente superior al DMSO en la congelación convencional de semen de $B$. henni. Por otro lado, Vuthiphandchai et al. (2009) y Rani et al. (2016) reportan altos porcentajes de vitalidad posdescongelación de semen criopreservado de Lutjanus argentimaculatus y Pangasius sutchi, respectivamente, empleando DMSO. Lo anterior muestra el uso común del DMSO y su efectividad en los procesos de criopreservación de semen de especies piscícolas; no obstante, en el presente estudio se demuestra la superioridad del EG en la conservación de la vitalidad del semen de Brycon henni. La Figura 1 muestra como las curvas de congelación ultra-rápida y media presentaron valores de VE superiores con el uso de ambos crioprotectores permeables, pero en todos los casos se observó una leve superioridad de los tratamientos de criopreservación suplementados con EG.

\section{Conclusiones}

- La criopreservación de semen de sabaleta (Brycon henni) por congelación programable, mediante una curva de descenso de temperatura de velocidad media (43.3 $\mathrm{min},-0.6^{\circ} \mathrm{C} / \mathrm{min}$ ), provee resultados superiores de calidad seminal posdescongelación.

- El uso de etilenglicol como crioprotector permeable en la congelación programable de semen de sabaleta (B. henni) permite alcanzar resultados superiores de vitalidad espermática posdescongelación. 


\section{Literatura Citada}

1. Arboleda L, Olivera-Angel M, Tabares CJ, Echeverrri A, Serna DF. 2005. Maduración gonadal en hembras de sabaleta Brycon henni y su relación con variables medioambientales. Rev Politécnica 1:95-103.

2. Atencio V, Perez, E, Pardo S. 2013. Evaluación de dimetilacetamida como crioprotector para la criopreservación de semen de bocachico (Prochilodus magdalenae). Arch Med Vet 45: 151158. doi: 10.4067/S0301-732X2013000200006

3. Babiak I, Glogowski J, Goryczko K, Dobosz, S, Kuzminski H, Strzezek J, Demianowicz W. 2001. Effect of extender composition and equilibration time on fertilization ability and enzymatic activity of rainbow trout cryopreserved spermatozoa. Theriogenology 56: 177-192. doi: 10.1016/S0093-691X(01)00553-2

4. Carneiro P, Azevedo, Santos J, Maria A. 2012. Cryopreservation of tambaqui (Colossoma macropomum) semen: extenders, cryoprotectants, dilution ratios and freezing methods. Cryo Letters 33: 385-393.

5. Carolsfeld J, Godinho H, ZaniboniFilho E, Harvey B. 2003. Cryopreservation of sperm in Brazilian migratory fish conservation. J Fish Biol 63: 472-189. doi: 10.1046/j.10958649.2003.00170.x

6. Cruz-Casallas P, Medina V, Velasco Y. 2006. Evaluación de diferentes crioprotectores para la crioconservación de espermatozoides de yamú Brycon amazonicus. Rev Colom Cien Pec 19: 153-159.

7. Gaitan-Espitia J, Martinez-Silva M, Borrero C, Ramirez L, Valencia J. 2013. Cryogenic preservation of sperm from lane snapper (Lutjanus synagris): testing the effects of extenders and freezing rates on sperm quality. Aquaculture 384-387: 6-12. doi: 10.1016/ j.aquaculture.2012.12.002
8. Gamboa S, Rodrigues A, Henriques l, Batista, C, Ramalho-Santos J. 2010. Seasonal functional relevance of sperm characteristics in equine spermatozoa. Theriogenology 73: 950-958. doi: 10.1016/j.theriogenology.2009.11.023

9. Hossain M, Sarder M. 2009. Cryogenic freezing of silver carp spermatozoa for conservation of gene pool. Progress Agric 20(1-2): 99-106. doi: 10.3329/pa.v20i1-2.16861

10. [ICA] Instituto Colombiano Agropecuario. 2007. Buenas prácticas en la producción acuícola: directrices sanitarias y de inocuidad para la producción acuícola destinada al consumo humano. Bogotá: Produmedios. 65 p. [ICA]. Disponible en: http://www.cib.uaem.mx/pdf/buenas_practicas.pdf

11. Lenis $G$, Restrepo L, Rivera $J$, Monsalve F, Cruz-Casallas P. 2009. Reproducción inducida y producción de alevinos de sabaleta Brycon henni: determinación del tiempo de latencia utilizando extracto de hipófisis de carpa. Rev Colom Cien Pec 22: 143-155.

12. Maria A, Viveiros A, Freitas $R$, Oliveira A. 2006. Extenders and cryoprotectants for cooling and freezing of piracanjuba (Brycon orbignyanus) semen, an endangered Brazilian teleost fish. Aquaculture 260: 298-306. doi: 10.1016/j.aquaculture.2006.06.011

13. Martínez G, Atencio V, Pardo S. 2011. Efectos de la concentración de glucosa sobre la activación de la movilidad espermática en bocachico Prochilodus magdalenae (Pisces, Characiformes). Rev MVZ Córdoba 16: 2554-2563.

14. Martínez Orozco HJ, Vásquez Zapata G. 2001. Aspectos reproductivos de la sabaleta Brycon henni (Piscis: Characidae) en el embalse la Salvajina, Colombia. Rev Asoc Colomb Ictiol 4:75-82.

15. Martínez S. 2008. Bases para la elaboración de bancos de germoplasma en peces: aplicación a la trucha leonesa. Tesis Doctoral. León, España: Universidad de León. 174 p. 
16. Medina M, Velasco Y, Cruz-Casallas P. 2005. Aspectos generales de la crioconservación espermática de peces teleósteos. Rev Colom Cien Pec 18: 34-36.

17. Montoya A, Carrillo L, Olivera M. 2006. Algunos aspectos biológicos y del manejo en cautiverio de la sabaleta Brycon henni Eigenmann, 1913 (Pisces: Characidae) Rev Col Cienc Pec 19: 180-185.

18. Phronen J. 1994. Composition and cryopreservation of sperm from some Finnish teleost fish. Finnish Fish Res 15: 27-48.

19. Pineda-Santis H,Arboleda L, Echeverry A, Urcuqui S, Pareja D, Olivera M, Builes J. 2007. Caracterización de la diversidad genética en el pez Brycon henni (Characiformes: Characi-dae) en Colombia central por medio de marcadores RAPD. Rev Biol Trop 55: 10251035. doi: 10.15517/rbt.v55i3-4.5975

20. Pineda-Santis H, Gómez-Oquendo J, Montoya-Páez J, Toro-Rendón V, Acevedo-Villa O, Restrepo-Betancur G, Toro-Rendón V. 2015. Crioconservación de semen y calidad espermática en sabaleta Brycon henni
(Pisces: Characidae). Orinoquia 19: 176183.

21. Rani K, Dhanasekar K, Munuswamy N. 2016. Fertilizability of cryopreserved and cadaveric fish spermatozoa of freshwater catfish Pangasius sutchi (Fowler, 1937). Aquacul Res 47: 15111518. doi: 10.1111/are.12611

22. Rani K, Munuswamy N. 2014. Preliminary studies on the cryopreservation of spermatozoa in the fresh water fish common carp (Cyprinus carpio L). J Coastal Life Med 2: 181-186. doi: 10.12980/JCLM.2.2014C1254

23. Viveiros ATM, Godinho HP. 2009. Sperm quality and cryopreservation of Brazilian freshwater fish species: a review. Fish Physiol Biochem 35: 137150. doi: 10.1007/s10695-008-9240-3

24. Vuthiphandchai V, Chomphuthawach $S$, Nimrat S. 2009. Cryopreservation of red snapper (Lutjanus argentimaculatus) sperm: effect of cryoprotectans and cooling rates on sperm motility, sperm viability and fertilization capacity. Theriogenology 72: 129-138. doi: 10.1016/j.theriogenology.2009.02.013 